\title{
La didactique du français : questions d'enjeux et de méthodes
}

\section{Bertrand Daunay et Yves Reuter}

\section{(2) OpenEdition}

1 Journals

\section{Édition électronique}

URL : https://journals.openedition.org/pratiques/1152

DOI : 10.4000/pratiques. 1152

ISSN : 2425-2042

Éditeur

Centre de recherche sur les médiations (CREM)

\section{Édition imprimée}

Date de publication : 15 juin 2008

Pagination : 57-78

\section{Référence électronique}

Bertrand Daunay et Yves Reuter, «La didactique du français : questions d'enjeux et de méthodes », Pratiques [En ligne], 137-138 | 2008, mis en ligne le 15 juin 2008, consulté le 28 juin 2022. URL : http:// journals.openedition.org/pratiques/1152; DOI : https://doi.org/10.4000/pratiques. 1152

(C) Tous droits réservés 


\title{
La didactique du français : questions d'enjeux et de méthodes
}

\author{
Bertrand Daunay \\ Yves Reuter
}

Université Charles-de-Gaulle - Lille 3

Équipe Théodile (ÉA 1764)

\section{Introduction}

La didactique du français est une discipline de recherche encore récente, dont la stabilité institutionnelle n'est pas assurée et qui est encore largement ignorée en tant que discipline de recherche, y compris au sein des instances institutionnelles et professionnelles de l'enseignement. Si le succès du mot « didactique » a été assuré notamment par des ouvrages de formation et, en France, par les concours de recrutement, c'est au prix d'un double malentendu. Le premier était dénoncé par J.-F. Halté en 1992 (p. 5 sq.) : le didactique serait « entendu comme le repli sur les contenus », fruit de la montée en puissance du « camp conservateur », contre le « discours pédagogique » qui se cantonnerait aux moyens d'enseignement - ce qui, précise évidemment Halté, « donne une idée fausse de la didactique, et de la pédagogie $»$.

Le deuxième malentendu tient à une confusion des ordres de réflexion sur l'enseignement, la dimension praxéologique de la didactique (qu'a si bien défendue Halté) pouvant facilement être entendue à tort comme une mise en pratique de savoirs déjà là - issus de champs de recherche déjà constitués : c'est là un avatar de l'applicationnisme, que la didactique naissante a, paradoxalement, à la fois combattu et d'une certaine manière promu, notamment en introduisant des savoirs issus de disciplines nouvelles, sans prendre toujours le temps de les intégrer à son propre espace de réflexion avant qu'ils ne soient diffusés dans le champ pratique.

Ce deuxième malentendu se nourrit de deux confusions - que vise actuellement à éviter la recherche en didactique, mais auxquelles elle n'a pas toujours pris garde et qu'elle n'évite pas encore toujours - d'une part entre les savoirs (construits ou convoqués) pour la recherche didactique (qui ne sont pas censés quitter le cadre théorique où ils prennent sens), les savoirs pour l'enseignement 
(conçu comme outils et non comme objets d'enseignement) et les savoirs destinés à la classe (autrement dit les objets d'enseignement); d'autre part entre description et prescription, c'est-à-dire entre ce qui relève de la construction théorique destinée à rendre compte du « réel » ou du «possible» et ce qui relève de l'injonction, destinée à accompagner l'action effective.

Or la didactique du français, comme toutes les didactiques, comme d'ailleurs toutes les disciplines de recherche, ne peut éviter de se penser dans sa spécificité et donc dans son autonomie, sur les plans conceptuel et méthodologique, aussi bien par rapport aux autres disciplines qu'elle côtoie que par rapport au champ pratique qu'elle explore. Cette nécessité, de forte exigence, est source de tensions comme de difficultés - tant théoriques que pratiques d'ailleurs -, mais constitue la condition non seulement de son institutionnalisation, mais encore de sa visibilité, c'est-à-dire in fine de son utilité sociale.

C'est cette question de l'autonomie disciplinaire que nous nous proposons d'aborder ici, par un rapide parcours historique de la discipline et par un examen des objets comme des méthodes de la didactique du français.

Dans un premier temps, nous rappellerons que l'émergence de la didactique du français s'est faite sous le signe d'une double tension-qui peut aussi caractériser d'autres didactiques, mais sous d'autres formes - entre un engagement militant et une visée scientifique, entre une inscription dans la pratique et une exigence théorique. Cela permettra d'interroger la lente autonomisation de la didactique par rapport aux espaces du militantisme et de la prescription.

Nous interrogerons ensuite les méthodes de recherche en didactique du français, pour tenter d'en approcher les spécificités et les caractéristiques les plus marquantes et de faire ressortir quelques conséquences des choix méthodologiques de la discipline sur sa propre cohérence scientifique.

Enfin, nous évoquerons le dialogue qu'entretient la didactique avec quelques autres disciplines de recherche, pour montrer à la fois son intégration dans un champ scientifique plus large et ses apports spécifiques de connaissances scientifiques.

\section{Quelques remarques sur la constitution du champ des recherches en didactique du français ${ }^{(1)}$}

\subsection{Tension entre engagement militant et visée scientifique}

Le « champ didactique »s'est constitué au début (entre les années 1970 et 1990) sur un mode dominé, à la fois du fait des disciplines de référence où les recherches puisaient (qui étaient soit de faible légitimité - comme les sciences de l'éducation ou le FLE - soit de légitimité récente et discutée - comme la linguistique) et du fait que les acteurs (personnes ou institutions) étaient elles-mêmes dominés ou discutés (praticiens, INRP, par exemple).

Ce statut dominé des acteurs, des disciplines et des institutions était finalement l'une des clés de l'entreprise et pouvait être une revendication : dans un

(1) Pour un développement des réflexions de cette partie, $c f$. Daunay (2007b, pp. 20-27). 
contexte de contestation de l'institution scolaire, issu notamment de la lecture des théories sociologiques de la reproduction, mais aussi des contenus d'enseignement dérivés des bouleversements épistémologiques apportés tant en linguistique qu'en littérature, penser l'enseignement du français nécessitait « une formation spécifique au-delà de la formation académique classique » (Ropé, 1990 , p. 142) mais aussi, souvent, un positionnement en rupture avec les instances traditionnelles de légitimation de l'institution scolaire et un investissement de structures moins reconnues mais plus actives sur le plan pédagogique. Cela favorisait assurément, en lien avec les divers mouvements de contestation politique dans les années 1960, l'adoption d'une posture militante, dont portent trace les revues professionnelles nées à l'époque, organes d'associations qui s'ancrent résolument - et explicitement - dans le militantisme : Le Français aujourd'hui (1969), Bref (1970), Pratiques (1974)...

Ce militantisme - c'est encore un trait de l'époque - se fonde sur une exigence théorique : précisément, la contestation de l'ordre scolaire établi passait par une revendication de refondation de la matière « français » sur des bases théoriques nouvelles, par référence à d'autres savoirs que les savoirs de référence des concours de recrutement ou des programmes d'enseignement. Mais, et c'est ce que nous identifions ici comme source de tension entre engagement militant et visée scientifique, la dimension militante des approches de l'époque revendiquait en fait une transformation de l'enseignement, avant même sa description ou sa compréhension. En témoigne le souci constamment renouvelé de penser l'enseignement en termes de rénovation ou d'innovation, en critiquant - plus qu'en décrivant - les formes «traditionnelles » d'enseignement (qu'il s'agisse des contenus ou des méthodes).

De fait, la naissance de la didactique du français s'inscrit dans un «mouvement de rénovation », pour emprunter l'expression de J.-F. Halté (2002, p. 18), qui précisément, dans une approche sous forme de bilan de la didactique de l'écriture, emploie l'expression sans l'interroger, comme si elle s'était lexicalisée pour désigner une période bien identifiée de l'histoire de l'enseignement ${ }^{(2)}$, ce qui s'observe souvent dans les bilans didactiques. Mais il est clair que ce qui passe désormais comme un moyen de périodisation renvoie à ce qui fut d'abord un mot d'ordre militant.

Or une exigence scientifique ne saurait se satisfaire de cette posture a priori, comme en témoigne l'évolution de Pratiques, dont rend compte A. Petitjean (1998, p. 5), à l'occasion du changement de formule de la revue : Pratiques continuera bien «d'analyser l'ancienne configuration didactique, mais de façon plus historienne que polémique ». Cela reste néanmoins une interrogation au sein de la didactique : par exemple, C. Simard (2001, p. 37), constatant « 1'antitraditionalisme affiché par la didactique du français » se demande si la recherche ne gagnerait pas «à adopter un point de vue plus ouvert et plus explicatif face à la tradition $»$.

Il faut noter que ce double positionnement - militant et scientifique - de certains acteurs ayant contribué à la naissance de la didactique peut encore se justi-

(2) Il emploie à plusieurs reprises dans son article le terme de « rénovation» dans ce sens. Ainsi le «mouvement de rénovation » peut-il s'opposer à «l'ancienne pédagogie du français». 
fier aujourd'hui par le projet social que se donne parfois la didactique : la DFLM, par exemple, dans son texte d'orientation (qui date de 1998), préconise « une attention particulière aux savoirs et aux méthodologies qui permettent de combattre l'échec scolaire et social» (p. 29); et J.-F. Halté considérait que la didactique devait « influencer» et «transformer » les pratiques « dans la perspective de la lutte contre 1'échec scolaire » (2001, p. 14). C'est à ce titre que B. Schneuwly, dans ses notes sur l'histoire de la didactique (1990, p. 22), observe que, " dès son origine» (au XVII ${ }^{\mathrm{e}}$ siècle, notamment chez Comenius), didactica est « un terme de combat » qui « sert à désigner et à promouvoir une conception nouvelle, démocratique de l'école »(Schneuwly, 1990, p. 22); il ajoute en conclusion (p. 24) : «L'idéal démocratique de Comenius est toujours présent dans la discipline». Qu'une science ait un projet social n'est pas vraiment propre à la didactique... Mais on voit bien ce que sa revendication peut engendrer comme tension entre l'engagement militant qui le fonde et le positionnement scientifique qui le justifie.

Il convient enfin d'évoquer une autre dimension de la didactique : le rapport à l'institution, concrétisé par l'investissement de structures de formation et de recherche (en France : INRDP, puis MAFPEN et, plus tard encore, IUFM) qui inscrivaient le souci de rénovation des acteurs dans une attente institutionnelle. Cette inscription institutionnelle de l'engagement de certains acteurs pouvait d'ailleurs entrer en conflit avec une critique de l'institution, sur d'innombrables aspects (dont principalement les contenus des programmes, même rénovés). Mais ce qui compte est de noter que finalement le militantisme et l'institution se retrouvent sur le terrain de la prescription et de la recommandation, où le chercheur, a priori, évite de se situer ${ }^{(3)}$. Cette possible confusion des genres trouve sa source sans doute dans l'indétermination initiale des espaces de travail des acteurs de la recherche en didactique et perdure encore à certains égards, tant sur le plan institutionnel ${ }^{(4)}$ que dans la production éditoriale, où peuvent se côtoyer discours prescriptifs et discours descriptifs - lesquels n'échappent pas toujours à la dimension prescriptive.

\subsection{Tension entre exigence théorique et inscription dans la pratique}

Les considérations qui précèdent ont dessiné en creux une autre tension - entre une inscription dans la pratique et une exigence théorique : c'est là un trait constitutif de la didactique, qu'elle n'arrête pas, depuis son émergence, de penser. Certes, on l'a vu, cette tension entre théorie et pratique peut trouver son origine dans le projet militant de certains de ses acteurs : non seulement dans la volonté de collaboration entre praticiens et théoriciens, mais au nom d'un primat de la pratique, conforme aux visées de transformation portées par les militants. Mais, plus fondamentalement, la tension entre théorie et pratique tient à la visée praxéologique de la didactique, qui fait des pratiques la base comme la finalité de la recherche ( $c f$. Halté, 1992 ; Brassart, Reuter, 1992).

Cette visée praxéologique oblitère-t-elle la dimension scientifique de son

(3) Sur la distinction entre les espaces, $c f$. Reuter éd. (2007, pp. 69-70).

(4) Du fait qu'un grand nombre de chercheurs en didactique, formateurs par ailleurs, se trouvent impliqués dans le système de recrutement ou de promotion des enseignants. 
projet de connaissance ? Ce qui ressort ici est la question épistémologique de l'intervention dans le champ didactique. J.-P. Bronckart (2001) montre que « la problématique de l'intervention » sous-tend non seulement les didactiques mais les sciences de l'éducation de manière générale et plus encore les sciences humaines et sociales depuis leur constitution. Il n'était pas dit à l'avance, dans les débats épistémologiques du début du $\mathrm{XX}^{\mathrm{e}}$ siècle, que l'approche scientifique fondamentale devait se découpler du domaine de l'intervention : mais c'est bien ce qui s'est opéré finalement, et de façon croissante, pour maintenir aujourd'hui encore une dualité d'approche qui s'est traduite, entre autres, par cette conséquence (ibid., p. 136) :

Entre les sciences humaines fondamentales et le domaine de l'éducation, se sont établis les rapports hiérarchiques et descendants de l'applicationnisme : les données scientifiques étaient injectées dans le champ pratique, la plupart du temps sans réelle prise en compte des multiples paramètres qui régissent ce dernier.

Cela interroge le statut même de la didactique (et des autres champs des sciences de l'éducation) : sa visée praxéologique, qui lui est consubstantielle, peut l'amener, dans cette logique de partage des tâches entre recherche et intervention, à se définir comme ingénierie - à tendance applicationniste. Bronckart critique cette position pour suggérer une autre voie (ibid., p. 138) :

Si l'on considère que les savoirs, même savants, sont toujours discutables, que les processus de transmission sont complexes et problématiques, et que les enjeux sont en permanence à repenser à la lumière des évolutions réelles des sociétés, alors il y a place pour une science véritable, dont l'objet est constitué par les processus de médiation formative, tels qu'ils sont conçus, gérés et mis en place par les sociétés humaines.

En fait, pour Bronckart, la didactique peut participer au retour de la question de «l'agir humain dans le monde», qu'atteste le mouvement opéré dans diverses sciences humaines, notamment en sociologie, en linguistique, en psychologie, et qui interdit de couper les interrogations scientifiques fondamentales des « dimensions actives et interventionnelles des conduites humaines » (ibid., p. 136$)^{(5)}$.

Mais le caractère encore programmatique de cette déclaration suffit à comprendre que ce qui peut être pensé en harmonie sur le plan épistémologique a des chances d'être vécu sous forme de tension, quand s'affrontent encore des définitions de la didactique qui sont pensées comme antagonistes et non complémentaires : technologie vs recherche, ingénierie $v s$ comme science.

(5) C'est la problématique de l'agir qui amenait déjà Bronckart, en 1989 (p. 54), à définir la didactique comme « discipline d'action » ou comme « technologie », quand il envisageait, sans précisément établir de frontière entre les domaines, deux types d' "interventions » de la didactique : une «théorisation des pratiques » et une « modification des pratiques » (ibid., p. 59). C'est, semble-t-il, à cette logique qu'obéit la définition de la didactique par Halté (1992, p. 16), quand il considère la didactique comme « discipline théorico-pratique » : «Son objectif essentiel est de produire des argumentations "savantes", étayées et cohérentes, susceptibles d'orienter efficacement les pratiques d'enseignement. » ( $c f$. Halté, 2001, p. 14). 


\subsection{Vers une autonomisation}

Les tensions constitutives de la didactique dans sa phase d'émergence, telles que nous avons essayé de les décrire, sont-elles en voie de disparition dans la phase d'autonomisation actuelle du champ de la recherche ? Une telle autonomisation se voit notamment dans l'évolution de certaines revues, qui, vers la toute fin des années 1980 ou dans les années 1990, se sont dotées d'un comité de lecture ou scientifique, alors qu'elle étaient d'abord élaborées par un " comité de rédaction » ou un "collectif»: c'est le cas, par exemple, de Repères, de Pratiques et d'Enjeux. Il n'est pas anodin, par exemple, que Repères ait, au lancement de sa nouvelle série en 1990, changé de sous-titre : revue «Pour la rénovation de l'enseignement du français », elle devient ainsi revue de « Recherches en didactique du français langue maternelle »; il n'est pas anodin non plus que certaines d'entre elles se dotent d'un comité de lecture. C'est dans Pratiques que se tient le discours explicatif le plus clair sous la plume d'André Petitjean, dans son avantpropos à Pratiques n ${ }^{\circ}$ 97-98: «Pratiques a 25 ans ». Commentant le changement de posture personnelle et institutionnelle des rédacteurs, Petitjean (1998, pp. 35) décrit le passage d'une «position essentiellement critique par rapport aux savoirs enseignés » (position militante) à une posture (épistémologique celle-là) «d'assimilation critique de notre travail », tout en revendiquant encore le projet social fondateur de la didactique : «aider à résoudre les formes présentes de l'échec scolaire $\gg$.

Il est intéressant d'observer que, dans la déclaration de principe d'une revue qui veut contribuer à l'autonomisation du champ de la recherche en didactique, subsistent ou resurgissent des tensions épistémologiques que nous avons tenté de décrire comme constitutives de la didactique ${ }^{(6)}$. Du reste, si cette autonomisation croissante s'accompagne (sans qu'il soit sûr qu'elle en soit la cause ou l'effet) d'un certain cloisonnement des espaces de discours, elle n'empêche pas la coexistence, au sein du champ, de revues aux statuts divers (scientifiques, militantes, institutionnelles), entre lesquelles on observe de constantes références croisées ${ }^{(7)}$. Au point d'ailleurs que l'on peut se demander dans quelle mesure ce croisement des espaces n'est pas un trait structurel de la didactique du français (cf. Daunay, 2007a).

Ce trait structurel tiendrait au conflit de valeurs que peuvent vivre nombre de didacticiens : si l'autonomisation de la didactique comme discipline de recherche peut avoir des avantages (légitimation institutionnelle et clarification des enjeux et des logiques des discours), elle porte en elle un risque qui peut inquiéter les acteurs du champ : l'éloignement des questions des chercheurs de celles

(6) Ces deux tensions que nous décrivons sont en fait essentiellement le fruit d'une tension constitutive des sciences de l'éducation en général, mis en lumière par Hofstetter et Schneuwly (1998/2001, p. 13), entre les demandes sociales qui légitiment les recherches en éducation et la nécessaire indépendance qu'une recherche, qui se veut scientifique, doit revendiquer par rapport à toute demande d'efficacité.

(7) Cela d'ailleurs ne serait pas un trait propre aux didactiques : la dimension militante et institutionnelle concerne en fait l'ensemble des revues touchant à l'éducation, comme l'atteste un rapport concernant les périodiques de langue française qui «contribuent à diffuser les questions, les débats, les pratiques et les recherches relatifs à l'éducation » (Beillerot dir., 1999, p. 5). 
des praticiens, avec comme corollaires, sur le plan pratique, des difficultés accrues de collaboration, et sur le plan épistémologique l'occultation de savoirs issus de pratiques ( $c f$. Reuter, 1995/2005, p. 216 ; 2006a, p. 11).

Cette autonomisation - relative - par rapport aux espaces du militantisme et de la prescription s'accompagne d'ailleurs d'une autonomisation par rapport à d'autres disciplines de recherche, dont semblait dépendre la didactique à ses débuts, comme les sciences du langage et les études littéraires. Du reste, cette autonomisation n'est pas nécessairement identique selon les champs de la didactique du français (grammaire, littérature, orthographe, etc.) comme le fait apparaître la comparaison des approches méthodologiques respectives de ces champs ( $c f$. Lettre de l'AIRDF $\mathrm{n}^{\circ} 40$ ) : la dépendance aux disciplines désignées comme références peut ainsi apparaître plus grande en littérature, par exemple. Par ailleurs, il ne semble pas qu'il faille négliger que la didactique n'est pas autonome institutionnellement, puisqu'elle dépend de structures qui lui impriment leur marque - institutionnelle, théorique, méthodologique : UFR de lettres, de sciences du langage, de sciences de l'éducation, entre autres.

C'est sans doute une des raisons qui ont retardé le rapprochement des didactiques entre elles : mais une nouvelle donne pourrait apparaître avec le développement du projet de didactique comparée, qui interroge la didactique du français à la fois dans sa spécificité de didactique (autonome donc des disciplines connexes qui ont pu lui servir naguère de référence) et dans son interdépendance à l'égard des autres didactiques : il faut à cet égard reconnaître l'importance décisive - au moins en France et en Suisse - des sciences de l'éducation dans la reconnaissance des didactiques et dans leur possible dialogue (Mercier, Schubauer-Leoni et Sensevy, 2002, p. 7).

Ce processus d'autonomisation ou, pour mieux dire, de reconfiguration disciplinaire de la didactique du français, est un processus en cours, qui force aux clarifications théoriques et méthodologiques, que l'on observe actuellement dans le champ.

\section{Questions méthodologiques ${ }^{(8)}$}

\subsection{Découpage des objets de la didactique du français}

La question se pose de la possibilité d'un « découpage thématique » de la didactique du français, dont Françoise Ropé, en 1990 (p. 54 sq.), signalait la difficulté méthodologique. Cette difficulté demeure aujourd'hui, dans la mesure où un tel découpage thématique ne peut se fonder sur un consensus établi.

Cette question pourtant est lourde d'enjeux, en ce qu'elle engage des conceptions différentes de la didactique. En effet, un objet de recherche didactique n'est pas un donné en soi, mais prend son sens dans une configuration didactique, qui correspond à un mode de découpage des questions traitées et donne aux objets ainsi isolés une cohérence suffisante dans un cadre théorique identifiable.

Les objets de recherche spécifiques de la didactique du français se détermi-

(8) Pour un développement des réflexions de cette partie, $c f$. Daunay (2007b, pp. 151-172). 
nent selon trois sources importantes. La première est la (ou les) matière(s) d'enseignement, qui indui(sen) ${ }^{(9)} 1^{\prime}$ 'identification d'objets ayant pris une valeur sociale indéniable : l'orthographe, par exemple, se laisse appréhender comme une partie spécifique de la matière français, non par son autonomie « en soi » (ce ne pourrait être qu'un sous-ensemble de l'étude de la langue, non identifiée comme telle), mais par le fait qu'elle soit socialement identifiable comme matière incontournable : ce qui suffit à en faire un objet didactique. De ce point de vue, le découpage qui fut longtemps dominant dans les instructions officielles françaises détermine des objets spécifiques : lecture, écriture, orthographe, grammaire pour le primaire et le premier cycle du secondaire; langue et littérature pour le lycée. Les modifications successives peuvent également donner lieu à l'identification d'objets didactiques, que ce soit par l'ajout d'un objet d'enseignement (l'oral, par exemple, depuis les années 1970-1980), par la spécification d'objets à enseigner ( $c f$. les « objets d'étude » dans les derniers programmes de français pour le lycée en France), par le regroupement d'objets (1'orthographe et la grammaire sont conçues actuellement comme sous-parties des « outils de la langue » au collège), par l'isolement d'un élément (la littérature au primaire comme discipline) ou encore par l'extension de ce qui fut préalablement identifié comme propre au cours de français (les «activités langagières » dans les disciplines, par exemple). La matière d'enseignement est encore une source possible de détermination d'objets didactiques quand est en jeu la compréhension des spécificités de chaque niveau scolaire, fixés institutionnellement, ou bien les dispositifs didactiques qu'elle promeut (comme les exercices, les activités, les modes de cloisonnement ou de décloisonnement des savoirs et des activités, etc.).

La deuxième source du découpage des objets didactiques se trouve dans les disciplines de référence traditionnelles de la didactique, dont la multiplicité (et parfois les conflits théoriques qui les opposent ou qui, au sein de certaines d'entre elles, opposent des écoles diverses) explique l' « apparition » d'objets didactiques propres qui n'entrent pas nécessairement en relation avec les objets traditionnels de la matière enseignée. L'importance prise par le « récit » par exemple, s'explique en grande partie par le développement des travaux de narratologie en littérature. C'est aussi le développement des théories littéraires qui peut expliquer que la « littérature », source quasi naturelle (c'est-à-dire impensée) des textes à lire autrefois, devienne un objet identifiable comme tel et donne à penser la possibilité d'une « didactique de la littérature ». C'est encore une discipline de référence, la psycholinguistique, qui permet de distinguer des objets didactiques identifiables, comme par exemple la compréhension et le déchiffrage en lecture ou encore la notion de « compétence métalinguistique ». Parfois, ce peut être la combinaison de plusieurs disciplines qui détermine un objet didactique : ainsi, le croisement de la linguistique, des théories littéraires et de la psychologie développementale explique l'« apparition » du genre en didactique.

La troisième source, enfin, est la tradition didactique elle-même, qui choisit ses disciplines de référence et opte donc pour un découpage de ses objets selon

(9) Ce pluriel de prudence s'explique par le fait, évoqué à l'instant, que la traditionnelle «matière » français peut éclater. Du reste, cela n'est peut-être que provisoire et il est par ailleurs fallacieux de parler d'une matière traditionnelle, tant ses contours ont varié dans 1'histoire, subissant le destin ordinaire des matières scolaires ( $c f$. Chervel, 1988/1998; 2006). 
des emprunts que l'histoire de la discipline peut aisément expliquer. Mais surtout, son souci de comprendre ce qui n'était pas objet d'une réflexion propre à des disciplines constituées explique 1'《 apparition »d'objets nouveaux, soit par la spécification d'objets non traités isolément ailleurs (1' "écriture créative » en est un bon exemple), soit par la création d'objets spécifiques (comme les interactions écriture-lecture ou les dispositifs didactiques concernant la lecture et l'écriture), soit par le regroupement d'objets traités ailleurs séparément (la « lecture ", par exemple, quand elle est conçue dans la continuité de son apprentissage premier et de son apprentissage continué de la maternelle à l'université, ou comme activité concernant la littérature et la non-littérature, voire dépassant le cadre de la matière " français »). C'est encore au sein de la didactique que peut prendre sens la modélisation de séquences didactiques ou l'analyse de situations didactiques que la discipline peut formaliser, par emprunt au cadre théorique d'autres didactiques ou par construction de modèles propres.

Ces trois sources de détermination des objets didactiques ${ }^{(10)}$ sont assez hétérogènes, ne sont pas nécessairement concordantes entre elles et ne présentent pas non plus chacune une absolue cohérence... Cela explique la diversité des objets, dont aucun classement général «objectif» ne saurait être effectué sans que soit perdue l'intention de leurs concepteurs ou la logique de leur conception. De ce fait, toute décision de découpage a posteriori ou toute insertion d'une recherche dans une classe donnée relève d'une décision qui n'est pas neutre épistémologiquement - et toute naturalisation de ces classements est de ce fait suspecte.

Une position « forte » concernant la didactique du français serait de considérer que l'unité des recherches est possible si les objets didactiques sont pensés et traités dans une didactique du français conçue comme discipline unifiée - et non comme agrégat de sous-disciplines : didactique de la littérature, de l'oral, de la français, etc. C'est, nous semble-t-il, la seule position épistémologique qui puisse donner à la didactique du français les moyens de traiter des questions d'enseignement et d'apprentissages des discours dans leur complexité, à tous les niveaux d'enseignement : cette conviction n'a cessé d'habiter J.-F. Halté dans sa volonté de penser la cohérence de la « matrice disciplinaire » du « français-matière » ${ }^{(11)}$, dont la didactique du français pouvait se donner, à ses yeux, comme discipline de référence ${ }^{(12)}$.

L'avenir de la didactique du français comme champ autonome de recherche, susceptible d'apporter des connaissances spécifiques, est au prix de cette reconnaissance de cette unité programmatique que les pionniers de la discipline avaient pensée, même s'il est nécessaire de la refonder régulièrement.

(10) Qu'il serait intéressant d'articuler avec l'identification que faisait J.-F. Halté (2001) des trois sources de savoirs (savants, sociaux ou socio-culturels, scolaires) où peut puiser la didactique.

(11) L'emploi de l'expression de Kuhn, fréquente chez Halté (notamment en 1992), est métaphorique, comme il le rappelle lui-même en 2001 (p. 17).

(12) 1992 et 2001, notamment. La conscience qu'avait Halté du danger de l'éclatement de la didactique en sous-disciplines explique que, dans un de ses derniers textes il ait tenu (certainement dans un souci polémique, comme le souligne É. Nonnon, 2006, p. 226) à rappeler les liens entre l'oral et l'écrit pour éviter qu'un champ en voie de constitution, la didactique de l'oral, en oublie la cohérence de la discipline (Halté, 2005). 


\subsection{Questions de méthodes}

Ce n'est pas seulement le choix des objets et de leurs configurations qui peut engager l'avenir du champ : une autre condition à la vitalité de la didactique comme discipline de recherche réside dans la clarification de ses méthodologies. Il est vrai que la question des types de recherche en didactique est constamment posée par les didacticiens dans leur quête de définition de la discipline, pour interroger sa légitimité « scientifique », sa cohérence épistémologique et méthodologique, son degré d'autonomie, ses relations à d'autres disciplines de recherche, les pratiques de ses acteurs, la validité de ses apports en termes de connaissances ou de méthodologie, son histoire et son devenir...

De multiples positions ont été prises sur cette question, notamment dans des publications collectives, depuis le numéro « historique » de Langue française $\mathrm{n}^{\circ} 82$ (1989), Vers une didactique du français?, en passant par les actes du colloque Questions d'épistémologie en didactique du français (Marquilló Larruy éd., 2001), jusqu'au dossier de la Lettre de l'AIRDF n 40 (2007), Les méthodes de recherche en didactique du français ${ }^{(13)}$. Ces interrogations prennent place dans un débat entre les didactiques, dans une perspective comparatiste, dont témoignent les récents séminaires internationaux sur les méthodes de recherche en didactique (Perrin-Glorian, Reuter dir., 2006 ; Lahanier-Reuter, Roditi dir., 2007).

Sans nous risquer ici à une synthèse, on peut s'en tenir au niveau de généralité de l'inventaire raisonné des types de recherches en didactique du français que constitue la banque de données DAF (Gagné, Lazure, Sprenger-Charolles, Ropé, 1989), qui est encore aujourd'hui une référence opérationnelle pour identifier les recherches en didactique du français, pour faire quelques remarques (trop rapides) sur quelques aspects saillants des questions de méthode qui peuvent se poser au sein du champ ${ }^{(14)}$.

Commençons par interroger la possibilité de l'expérimentation en didactique, ce qui suppose au préalable que l'on ait renoncé à croire possible de se référer à Karl Popper : Jean-Claude Passeron (1991/2006) a assez clairement fait justice de cette illusion dans un espace non-poppérien du raisonnement naturel (c'est le sous-titre de la première édition de son ouvrage). Parler de recherche expérimentale en didactique, ce n'est pas supposer mécaniquement transférable dans le champ le raisonnement expérimental qu'autorisent les sciences de la nature.

Suffit à établir cette différence de nature la dimension praxéologique de la didactique, qui l'empêche de se constituer en discipline de laboratoire, sauf à dénaturer son programme scientifique ( $c f$. Rosier, 2002, p. 105). Eddy Roulet constatait en 1989 (p. 6) :

(13) Si les questions de méthodes sont constamment posées par la didactique depuis ses débuts, on assiste à un réel développement de cette réflexion aujourd'hui ; on peut mesurer cette prise en compte croissante de la réflexion méthodologique par la comparaison entre les deux «Que sais-je » sur la didactique du français : dans celui qu’a rédigé J.-F. Halté (1992), elle n'a pas sa place, alors que dans celui qu'a rédigé J.-M. Rosier (2002), plusieurs pages lui sont consacrées.

(14) Les auteurs isolent quatre grands types de recherches : la recherche descriptive, la recherche expérimentale, la recherche-action, la recherche théorique. C'est à cette classification que nous nous référons dans l'usage de ces expressions. 
Il ne paraît guère réaliste, en l'état [...], d'imposer une validation expérimentale rigoureuse et reproductible. Certes, celle-ci est possible en didactique [...], mais au prix d'une telle réduction de l'objet d'étude, pour maîtriser toutes les variables, qu'elle limite drastiquement la portée des résultats obtenus.

C'est le problème qu'ont pu rencontrer les psychologies expérimentales, comme l'écrit Jean-Claude Passeron (1991/2006, p. 228 sq.) : elles «ont côtoyé au plus près la contradiction entre la pureté expérimentale et la portée générale de leurs assertions scientifiques »; de fait, l'adoption de «conditions formelles d'une application en laboratoire de la méthode expérimentale » s'est faite « au prix d'une miniaturisation de leurs objets d'observation », ce qui oblige les expérimentateurs eux-mêmes à interroger la «représentativité » des situations expérimentales par rapport à l'ensemble des situations habituelles. Passeron conclut : «Certains diront se contenter d'une validité de principe, fût-elle limitée aux situations improbables ou artificielles. Tout est là. »

Dès lors que le laboratoire n'est pas le lieu d'exercice du didacticien et que l'on reconnaît les contraintes qu'impose à la discipline sa dimension praxéologique, ce n'est pas renoncer au caractère scientifique de la discipline, mais c'est interroger la nature de cette scientificité. Il n'est pas sûr, par exemple, que la recherche-action et la recherche expérimentale en didactique reposent sur deux paradigmes différents de la recherche ( $c f$. Roulet, 1989, p. 5 sq. ; Dabène, 1999, p. 38). Ce qu'elles ont en commun, c'est l'ambition de mettre à l'épreuve empirique des propositions théoriques - quelque degré de légitimité théorique qu'elles aient par ailleurs - sans pour autant se croire tenu par le régime de la preuve.

L'une comme l'autre de ces recherches, qui sont toutes deux des formes d'expérimentation, peuvent apporter des connaissances, à deux conditions - qui paraissent des critères d'évaluation pertinents sur ce point : qu'elles s'articulent aux connaissances déjà acquises dans le champ (ce qui les adosse d'une manière ou d'une autre aux autres recherches, expérimentales ou non : c'est là une exigence théorique) et qu'elles ne sombrent pas dans la prescription (ce qui les oblige à la modestie non feinte de la délimitation la plus précise possible de leur portée - ou, pour ainsi dire, de leur empan de validité : c'est à soi seul une exigence de rigueur méthodologique).

La question de la légitimité des formes d'expérimentation en didactique du français semble souvent entaché de la recherche d'une légitimité "scientifique », comme s'il existait un brevet de scientificité accordé quasiment automatiquement par l'adoption d'une méthode ( $c f$. encore Passeron, 1991/2006, p. $228 \mathrm{sq}$.). La recherche de légitimité scientifique doit au contraire, pour être vraiment scientifique, se passer de fausses attestations. Et « si l'on tient à ce mot», comme le dit ironiquement Jean-Louis Chiss, la scientificité de la didactique ne fait pas tout : encore doit-elle interroger sa spécificité (Chiss, 1989, p. 51). À ce titre, ce sont ses objets qui l'amènent - progressivement - à déterminer ses méthodes : et c'est là tout un programme scientifique... Pour Jean-Louis Chiss (ibid.), si l'on tient à viser la scientificité en didactique, c'est là une « affaire d'explicitation, de clarification, de classement $»$.

Cela explique sans doute le développement, en didactique du français, de ce que Gagné, Lazure, Sprenger-Charolles, Ropé (1989) appellent la recherche descriptive; discutant la distinction parfois difficile entre recherche descriptive 
et recherche expérimentale, les auteurs les différencient notamment par le fait que la recherche descriptive ne manipule pas mais observe des variables. C'est d'ailleurs peut-être là, au confluent de deux méthodes, que se trouvera une spécificité des méthodes de recherche en didactique, si l'on en croit Mercier, Schubauer-Léoni, Gérard Sensevy (2002, p. 11) :

Une partie des chercheurs qui, à ce jour, se sont engagés dans un projet de didactique comparée, situent leur effort de clarification méthodologique à l'articulation de deux versants qui tendent habituellement à s'exclure : le clinique et l'expérimental.

Une autre forme de recherche particulièrement importante dans le champ est la recherche théorique ${ }^{(15)}$, pour laquelle il est difficile de repérer des constantes de méthode. Non que les recherche de ce type manquent de rigueur : même quand elles sont rigoureuses dans l'examen des données (théoriques ou empiriques) et dans l'argumentation, elles ne sauraient être évaluées selon les critères d'une méthodologie suffisamment unifiée pour servir de référence.

C'est du reste une des limites des recherches théoriques en didactique du français, dans la mesure où elles ne font pas vraiment l'objet d'une réflexion collective sur la nature des méthodes, réflexion qui en permettrait une réelle évaluation ${ }^{(16)}$. Par ailleurs, l'importance en nombre des recherches théoriques en didactique du français peut ne pas tenir à des choix scientifiques raisonnés qui en légitimeraient l'usage, mais à une sorte d'habitude du travail intellectuel dans la communauté des chercheurs concernés ( $c f$. Goigoux, 2001, p. 126). Le risque est grand que de telles recherches passent assez facilement pour naturelles aux yeux de chercheurs dont l'histoire personnelle peut parfois aisément les cantonner dans un modèle discursif de nature philosophique ou littéraire, avec lequel l'étude théorique a des affinités.

Un autre risque des études théoriques peut être de donner une place plus importante qu'il ne faut à l'application théorique d'éléments disciplinaires, l'une des démarches isolées par Gagné, Lazure, Sprenger-Charolles, Ropé (1989, p. 58). C'est une des explications que donnent Daunay, Dufays (2007, p. 12) à l'importance des recherches théoriques concernant la littérature. Ce qui va contre le «mouvement ascendant et non descendant » que préconisait J.P. Bronckart (1989, p. 64) dans sa conception d'une didactique autonome, inscrite dans le champ de « la science de l'éducation».

Moyennant une vigilance à l'égard de ces effets pervers, les recherches théoriques semblent cependant indispensables à la recherche didactique, en ce qu'elles lui permettent de penser ses objets : interroger constamment ce que les traditions diverses qui traversent le champ (que ce soit la tradition de la discipline elle-même ou celle des disciplines contributoires comme encore celle des matières d'enseignement) ont tendance à facilement naturaliser peut aider à éviter tout aveuglement épistémologique.

(15) Pour reprendre encore la terminologie de Gagné, Lazure, Sprenger-Charolles, Ropé (1989). Ce type de recherche gagnerait cependant à être plus strictement définie.

(16) Il n'est pas sûr par ailleurs que cette réflexion sur ce que nous entendons ici par recherche théorique soit vraiment plus poussée dans les autres disciplines. 
Exemplaire à cet égard est le développement de l'étude historique ${ }^{(17)}$. Ce développement s'explique à la fois par la maturation scientifique du champ ( $c f$. Petitjean, Privat, 1999) et par la volonté de promouvoir, dans le champ de la didactique du français, une réflexion davantage centrée sur la compréhension des phénomènes didactiques (contextualisés dans leur histoire) que sur la dénonciation du passé, qui a pu caractériser les premiers travaux didactiques.

Il nous semble important de terminer cette réflexion sur les méthodes de recherche en précisant qu'elles concourent à la compréhension des phénomènes didactiques et peuvent être, in fine, source de décisions politico-institutionnelles en matière d'éducation, mais ne sauraient se confondre avec elles. Le programme scientifique de la didactique exige en quelque sorte la construction d'une intelligibilité du réel qui ne prétende pas peser sur lui. Certes, on peut considérer que la finalité ultime de la recherche didactique est l'amélioration de l'enseignement ; mais ce possible usage social de la didactique concerne le champ dans son ensemble et non telle ou telle recherche didactique spécifique, qui doit au contraire renoncer à postuler une efficacité directe et immédiate pour satisfaire aux exigences méthodologiques et épistémologiques d'une recherche scientifique.

On retrouve là une tension constitutive de la didactique et de toute science de l'éducation, telle que Hofstetter et Schneuwly (1998/2001, p. 13) la décrivent, entre la « réponse à de fortes demandes sociales du terrain et de la sphère politico-administrative » et « la construction d'une discipline scientifique pluridisciplinaire ». Or cette dernière postule une «suspension de l'action » (ibid.). Il nous semble que la didactique du français n'est pas encore totalement au clair avec ces problèmes et que la confusion règne trop souvent entre la finalité sociale ultime de la recherche didactique - qui est de nature collective et différée - et la possibilité d'une efficacité immédiate et ponctuelle de telle ou telle recherche.

C'est que la dimension normative et prescriptive de bon nombre de recherches, qu'expliquent assez facilement l'histoire de la discipline et le positionnement institutionnel de ses acteurs, aveugle parfois l'épistémologie du chercheur. Certes, comme le dit M. Bru (1998, p. 54), « les obstacles de l'orientation prescriptive ne condamnent pas les sciences de l'éducation à abandonner toute mise en relation de recherches et des pratiques ». Mais, ajoute-t-il, «il reste à modéliser ces relations sans les réduire à une visée applicationniste ou à une version idéalisée, étrangères à ce que sont les pratiques en situation. » Or force est de constater que les orientations prescriptive et descriptive, qui relèvent d'espaces institutionnels, professionnels, discursifs différents, sont parfois confondues au sein même de recherches descriptives ou théoriques en didactique du français.

Rien n'empêche un didacticien de concevoir des outils d'enseignement, voire de les légitimer par des emprunts aux savoirs théoriques construits dans ses propres recherches ou dans celle du champ. Il y a en revanche confusion des genres - et elle est à nos yeux problématique - quand, au sein même de recherches descriptives ou théoriques, le chercheur construit un modèle en le supposant applicable sans analyser précisément les conditions de cette « applicabilité » - ana-

(17) Que Gagné, Lazure, Sprenger-Charolles, Ropé (1989, p. 47), pour leur part, incluent parmi les recherches descriptives. 
lyse risquée, il est vrai, en ce qu'elle l'amènerait vraisemblablement à abandonner quasiment tout le temps cette prétention...

Plus problématique encore est à nos yeux la posture d'évaluation prise parfois quand il s'agit de pratiques d'enseignement : maintes communications en didactique du français négligent la distinction entre une évaluation théorique, qui interroge, aussi objectivement que possible, une situation didactique et un jugement de valeur qui ne fait que dévoiler des conceptions propres au chercheur, dont rien ne dit qu'un examen attentif ne les ramènerait pas au rang de simple opinion (cf. Daunay, 2007a).

Il y a là un risque pour la didactique, dans la mesure où la confusion entre prescriptif et descriptif peut justifier finalement que les propositions théoriques soient immédiatement mesurables en terme d'efficacité pratique : on sait le parti que peuvent tirer les adversaires de la discipline de cette exigence... C'est précisément pour cela qu'une autonomie de la didactique comme discipline de recherche nous semble nécessaire, ce qui l'oblige à un retrait par rapport à son usage possible-lequel, en même temps, ne peut pas ne pas être pensé au sein même des recherches en didactique.

\section{Dialogues avec d'autres disciplines}

\subsection{Fragilités de la didactique du français}

L'autonomisation de la didactique du français - par rapport aux espaces institutionnels et aux autres disciplines de recherche - pourrait supposer qu'elle manifeste qu'elle est capable d'apporter des connaissances que d'autres disciplines ne permettent pas de construire dans leur cadre théorique. Cela demanderait que les frontières entre les disciplines soient plus nettement marquées ou tout au moins que les autres disciplines identifient la didactique comme une discipline sœur, susceptible de participer à un dialogue avec elle.

Cela n'est pas encore totalement réalisé. L'exemple de la sociologie est à cet égard assez parlant. Son influence fut considérable sur la didactique du français ou plus exactement sur la conscience de la nécessité d'une didactique du français, discipline destinée à penser l'enseignement autrement que dans son «fonctionnement au talent et au don », comme le dit Halté (1987, p. 93), en référence directe aux travaux de Bourdieu et Passeron, dont on pouvait penser qu'ils décrivaient clairement la «configuration ancienne » de l'enseignement du français ( $c f$. les références explicites et récurrentes à ces auteurs dans Halté, 1992). Pourtant, au début des années 1990, quand des sociologues posent la question d'une approche spécifique des disciplines scolaires - qui est bien le programme des didactiques des disciplines...-, les références aux recherches didactiques ne s'imposent pas.

Ainsi, J.-C. Passeron (1991/2006), dont l'approche a évolué de la revendication d'une «pédagogie scientifique »-qui néglige la question des disciplines scolaires - (Bourdieu et Passeron, 1964, p. 111 sq.) à la formalisation très poussée d'une « didactique de discipline » (1991/2006, p. 532), que les didactiques pourraient, en la discutant, reprendre sans difficulté à leur compte, ne cite aucune source didactique comme possible influence de l'évolution de sa réflexion, 
même dans la réédition toute récente de son ouvrage... De même, quand B. Lahire (1993) infléchit les recherches sociologiques sur l' "échec scolaire» en centrant son approche sur la discipline « français » et ses sous-matières, dont il veut précisément interroger la " cohérence » (cf. 1993, p. 62), il ne recourt à aucune référence didactique, alors que son information est sérieuse, notamment par la prise en compte de disciplines théoriques qui influencent alors nettement la didactique (linguistique, psychologie sociale, histoire, anthropologie, théorie littéraire...), comme si la didactique, dans ses emprunts théoriques à ces mêmes disciplines, n'avaient rien apporté à la compréhension spécifique du fonctionnement des disciplines ${ }^{(18)} \ldots$

Sans doute une analyse sociologique permettrait de comprendre en partie ce caractère inaudible de la didactique... Mais il faut aussi s'interroger sur ses causes plus internes. Force est de constater, par exemple, que les apports de Bernard Lahire à la compréhension des phénomènes scolaires propres à la discipline « français » sont allés parfois plus loin que ce que certaines recherches didactiques ont pu apporter dans la caractérisation des sources d' "échec scolaire »en français : apparaît là une question épistémologique importante, qui est de savoir si l'entrée spécifiquement didactique dans l'analyse des phénomènes scolaires est susceptible de donner lieu à des apports de connaissances plus précis qu'une approche sociologique (ou psychologique, ou anthropologique...) « ciblée » peut permettre. Poser cette question, qui rejoint celle des frontières disciplinaires, est nécessaire à la caractérisation de notre discipline.

Par ailleurs, le nécessaire emprunt de la didactique du français à d'autres disciplines, dans sa phase d'émergence, a pu la contraindre dans son développement comme discipline autonome. Ainsi, pour quitter la référence à la sociologie et sans revenir aux tentations applicationnistes des débuts (par la reprise parfois non spécifiée de concepts ou de méthodes aux théories linguistiques ou littéraires), il n'est pas toujours sûr que les frontières entre description linguistique et description didactique soient fermes, notamment dans l'analyse des textes d'enfants ou des interactions en classe : dans ces exemples, si la problématique est spécifiquement didactique (il s'agit d'interroger les pratiques langagières induites par le système didactique), les méthodes d'analyse peuvent ne pas l'être. De même, les démarches empruntées à d'autres disciplines (psychologie du travail, ergonomie...) dans l'analyse du travail enseignant ne font pas toujours l'objet d'une interrogation épistémologique ou d'une spécification méthodologique. Autre exemple : la notion de « sujet», qui prend ces dernières années une importance croissante en didactique, n'est pas toujours pensée d'un strict point

(18) Il faut du reste à la fois généraliser et relativiser cette sorte d'ostracisme. Du côté de la généralisation, observons que la sociologie n'est pas la seule à être sourde aux apports de la didactique : par exemple, si A. Chervel, une référence majeure des recherches en didactique - depuis sa phase d'émergence jusqu'à aujourd'hui - emprunte volontiers, dans sa somme récente (2006) le mot « didactique » pour désigner l'approche des savoirs disciplinaires, il semble que ce soit son seul emprunt aux acquis théoriques de la didactique du français. Pour relativiser cependant, notons que la didactique n'est pas la seule discipline à ne pas être reconnue dans ses apports théoriques : il est frappant, pour ne prendre qu'un seul exemple, que la réflexion importante du même Chervel sur la question de la discipline ne rencontre qu'un écho étouffé dans la réflexion sociologique sur la forme scolaire, quand Vincent et al. (1994, p. 17) le citent rapidement, pour surtout souligner qu' " il ne recourt pas assez aux travaux des sociologues pour pousser plus loin l'analyse »! 
de vue didactique : pour le dire autrement, le sujet didactique est souvent absent des réflexions didactiques sur le «sujet», qui semble davantage être appréhendé comme sujet sociologique psychologique ou pédagogique. Enfin, les emprunts théoriques aux autres didactiques ne sont pas toujours clairement délimités : si la notion de «transposition didactique», issue de la didactique des mathématiques, a fait l'objet de nombreux débats, du fait de l'interrogation qu'elle suscitait sur le savoir en jeu dans les différentes disciplines, certaines notions sont en revanche reprise sans véritable souci de spécification, comme celles de dévolution, par exemple.

Pour autant, si nous écrivons ces lignes, c'est précisément qu'il est possible de les écrire, c'est-à-dire que la didactique n'ignore pas ces questions, depuis longtemps, même si tous les travaux ne les prennent pas en compte : comparativement à d'autres disciplines de recherche, il faut noter l'importance des réflexions théoriques et méthodologiques (dont nous rendions compte dans notre deuxième partie) en didactique du français sur la question des emprunts et des spécifications didactiques de notions issues d'autres disciplines de recherche.

\subsection{Les apports de la didactique du français}

La didactique du français peut précisément gagner, sur les plans épistémologique et méthodologique, à dialoguer avec les autres disciplines de recherches pour montrer en quoi une approche didactique permet de penser les apports respectifs de certains auteurs, comme par exemple Vygotski (Schneuwly, 2007), Goody (Reuter, 2006b), Bakhtine (Jaubert, Rebière, Bernié, 2003). Il ne faut pas négliger non plus le rôle de la didactique dans le développement de la fortune récente de ces auteurs, par la focalisation sur certains aspects de ces théories que la didactique met mieux en lumière : c'est particulièrement le cas de Vygotski, grâce à la contribution de l'équipe de Genève à la redécouverte pluridisciplinaire de cet auteur depuis la fin des années 1970, mais c'est aussi le cas, pour ne citer que les auteurs emblématiques déjà cités, de Bakhtine (avec un recentrage des lectures sur la question du genre - non littéraire) ou de Goody (avec une interrogation sur le rôle de l'écrit dans la constitution de la forme scolaire).

Ces confrontations de la didactique à d'autres disciplines - quand le risque de l'applicationnisme est réellement évité et que la spécification didactique des concepts est rendue possible - constituent une source de grande richesse épistémologique. La question de la norme, par exemple, est un objet de réflexion dont peut s'emparer la didactique (Nonnon, 2000) qui trouve là un objet de recherche différencié de ce que peuvent en dire des discipline comme la linguistique ou la sociologie.

De surcroît, la structure pluriréférentielle de la didactique peut l'amener à avoir une dimension de confrontation méta-méthodologique par rapport à d'autres disciplines en interrogeant leurs modes de construction de certains « faits» : la didactique interroge par exemple de façon centrale la question du méta, présente dans de nombreuses disciplines (linguistique, psycholinguistique, sociologie...) sans être toujours méthodologiquement réellement pensée. Dès lors, cela ouvre la possibilité d'autres approches, notamment celles qui interrogent la pertinence même de la notion de distance cognitive, du rapport à l'écrit, de la po- 
sition du scripteur, etc. ( $c f$. Delcambre, Reuter, 2002 ; Daunay, 2002); ou encore celles qui questionnent les mots des élèves pour dire le méta.

La didactique peut aussi interroger les autres disciplines et explorer des pistes plus finement, en fonction de questions centrales pour elles et secondaires pour les autres. On peut, pour illustration, prendre trois exemples parlants :

- la définition des performances des élèves ;

- les relations entre performances et apprentissages disciplinaires ;

- les relations entre enseignement et apprentissages disciplinaires.

La définition des performances des élèves a été une vraie question théorique pour la didactique, depuis ses débuts, qu'il s'agisse de l'évaluation scolaire ou de l'évaluation en recherche. La question s'est particulièrement posée du fait d'une confrontation des formes d'évaluation scolaires à des contenus définis autrement par les didactiques. Par exemple, en didactique du français, l'introduction de la dimension textuelle dans l'approche des écrits (leur cohérence, leur organisation interne) a pu faire apparaître l'insuffisance des évaluations traditionnelles en termes de correction linguistique ou stylistique, voire morale. Mais cela a aussi interrogé la manière d'analyser les performances spécifiques ciblées et de préciser la légitimité du type de mesures prises et des modes d'interprétation par rapport au cadre théorique convoqué : par exemple, quand on cherche à analyser la maîtrise de la compétence textuelle des élèves, se pose crucialement la question de la définition de critères qui ne soient pas intuitifs et qui soient par ailleurs reproductibles.

De la même manière, on a pu voir évoluer l'évaluation quand ont été introduits des contenus moins valorisés par la tradition scolaire, censée être plus centrée sur les connaissances et les savoir-faire : quand on s'est intéressé, par exemple, aux compétences, aux rapports à, aux postures, aux gestes, etc., la question de l'évaluation s'est posée autrement. D'où l'évidence d'une nécessaire interrogation sur la mesure des performances, différente selon qu'il s'agit de savoirs ou de rapports à, par exemple.

Ont été également observés les effets que peut produire la médiation par les instruments d'évaluation (oraux ou écrits) : ne pas être capable de répondre correctement à un exercice d'évaluation ne dit pas forcément que le savoir ou le savoir-faire en jeu n'est pas acquis, mais peut simplement informer du fait que l'élève ne sait pas répondre à la question posée dans la situation d'évaluation proposée. Ou encore telle réussite ou tel échec à telle évaluation peut être considéré comme le signe que l'habitude est prise ou non de répondre à un type de question, ce qui ne préjuge rien de la maîtrise ou de la non maîtrise effective de ce qui est évalué sur le moment et encore moins dans l'avenir. Ce qui a pu faire naître des questions théoriques importantes comme celle des parts respectives de ce qui relève de la maîtrise de la situation d'évaluation proposée et de la maîtrise de l'objet évalué.

Les relations entre performances et apprentissages disciplinaires sont au cœur de la réflexion didactique. La question de la mesure temporelle des apprentissages, notamment, se pose. Peut-on juger qu'un apprentissage est effectué quand il est évalué tout de suite après une séquence d'enseignement-apprentissage, ou doit-on le mesurer à quelque distance - et laquelle? 
D'autres questions se sont posées quand on s'est rendu compte qu'il n'est pas toujours aisé de cerner l'objet de l'évaluation, donc l'apprentissage mesuré. Dans le cas d'un savoir linguistique, par exemple, juge-t-on réussi l'apprentissage quand le savoir peut être déclaré, quand il peut être utilisé à bon escient, quand le système des conceptions qu'il a pu heurter est modifié, quand il permet la réorganisation du système des savoirs dans lequel il s'insère ? Ou encore quand il est utilisé en dehors de la situation même d'évaluation, voire en dehors de l'école, dans une situation ordinaire où la mobilisation de ce savoir peut être nécessaire ? Là encore, se sont imposées des questions épistémologiques et méthodologiques sur le mesurable, en précisant l'écart entre le déclaratif et l'apprentissage effectivement mesuré, en interrogeant les modes de manifestation de l'apprentissage, en questionnant les situations de recueil classique (qui, même en recherche, s'apparentent souvent aux modes de recueil propres à l'évaluation scolaire) ou selon des modes différents, soit périscolaires (par exemple les entretiens avec des enfants dans l'école) soit extrascolaires (totalement hors du système scolaire).

Les relations entre enseignement et apprentissages disciplinaires ne sont pas moins centrales dans le projet scientifique des didactiques. Si la mesure du «bon » enseignement reste un horizon possible pour la didactique, elle ne saurait être pensée, didactiquement, sans référence aux effets en termes d'apprentissages effectués ou possibles des élèves. Si resurgissent là les questions de la mesure des performances, de la temporalité des apprentissages, de ce que dit une performance d'un apprentissage, apparaît en outre la question cruciale de la possibilité d'une relation entre les pôles du triangle didactique. Comme le disait Halté (2001, p. 13 sq.) de la didactique, «si elle se veut interventionniste, c'est bien du système des relations entre variables qu'elle doit traiter in fine». Seule la didactique est en mesure d'apporter des connaissances nouvelles sur les liens qui peuvent s'établir entre un savoir, l'enseignant et l'apprenant, ce qui peut se décliner de diverses manières. Quel savoir un enseignement peut construire chez l'élève ? Quels savoirs savants peuvent fonder un enseignement en vue d'un apprentissage ? Quelles relations un enseignement construit-il chez l'apprenant entre un savoir savant et un savoir appris ? Etc.

S'il est une question qui distingue assez clairement la pédagogie de la didactique, par exemple, c'est cette exigence de systémicité (Halté, 2001) que le triangle didactique gagne sur le triangle pédagogique. Mais, plus généralement, c'est sans doute dans l'affichage de cette exigence que la didactique du français peut se faire reconnaître des questionnements théoriques spécifiques, que ni la pédagogie, ni la sociologie, ni la psychologie, ni les diverses disciplines contributoires de la didactique du français n'ont vocation à traiter.

\section{Conclusion}

La didactique du français peut être aujourd'hui considérée comme une discipline consistante, si on mesure la consistance à la densité des questions théoriques nouvelles que pose une discipline aux «faits » empiriques. Rappelons à cet égard l'hommage que rendait D. Hameline (2005) aux didactiques : 
Phénomène démultiplié, imaginatif, parfois iconoclaste et flibustier, la saisie didacticienne a renouvelé profondément le regard pédagogique sur les savoirs et les compétences, que celles-ci soient disciplinaires ou transversales.

Si ce renouvellement a été possible, comme le suggère Hameline, c'est essentiellement en raison du regard critique qu'elle a porté sur les phénomènes scolaires, mais aussi sur ces propres enjeux et méthodes, ce dont cet article voulait témoigner. Cette dimension auto-méta-critique doit beaucoup, on le sait, à J.-F. Halté et à ses réflexions obstinées et incessantes sur les rapports entre didactique et disciplines contributoires (la linguistique et les études littéraires, particulièrement, pour Halté), entre didactique et pédagogie, entre engagement et scientificité, entre théorie et pratique. Ces questions, que nous avons voulu (re)parcourir à notre manière, ont été et demeurent fondamentales dans la détermination du programme scientifique de la didactique du français. C'est aussi bien par ses recherches empiriques que par sa contribution à la construction de ces questions, de ce programme et, conséquemment de notre discipline, que Jean-François Halté est, et restera, une référence incontournable.

\section{Références}

BEILLEROT, Jacky dir. (1999) : Les périodiques et l'éducation. Éléments pour un état des lieux de la diffusion de la recherche, Rapport pour le ministère de l'Éducation Nationale, de la recherche et de la technologie (Direction de la recherche, Comité national de coordination de la recherche en éducation), Paris, INRP.

Bourdieu, Pierre, PASSERon, Jean-Claude (1964) : Les Héritiers, Paris, Minuit.

BRASSART, Dominique Guy, REUTER, Yves (1992) : «Former des maîtres en français : éléments pour une didactique de la didactique du français », Études de linguistique appliquée, $\mathrm{n}^{\circ} 87$, Recherches en didactique du français et formation des enseignants, septembre 1992, Paris, Didier, pp. 11-24.

BRONCKART, Jean-Paul (1989) : " Du statut des didactiques des matières scolaires », Langue française $\mathrm{n}^{\circ}$ 82, Vers une didactique du français ?, mai 1989, Paris, Larousse, pp. 53-65.

- (2001) : "S'entendre pour agir et agir pour s'entendre », dans BAUDOUIN, J.-M., FRIEDRICH, J.éds., Théories de l'action et éducation, Bruxelles, De Boeck Université, pp. 133-154.

BRU, Marc (1998) : «Qu'y a-t-il à prouver, quand il s'agit d'éducation? La validation scientifique des propos et discours sur les pratiques d'enseignement : après les illusions perdues », in HADJI, C., BAILLÉ, J.éd., Recherche et éducation. Vers une "nouvelle alliance ». La démarche de preuve en 10 questions, Paris-Bruxelles, De Boeck, pp. 45-65. 
CHERVEL, André (1988/1998) : «L'histoire des disciplines scolaires. Réflexions sur un domaine de recherche », Histoire de l'éducation $\mathrm{n}^{\circ} 38$, Paris, INRP, pp. 59-119, repris dans La Culture scolaire. Une approche historique, Paris, Belin, 1998, pp. 9-56.

- (2006) : Histoire de l'enseignement du français du XVII au XX siècle, Paris, Retz.

CHISS, Jean-Louis (1989) : «Revendication d'autonomie et horizon de scientificité en didactique du français ", Langue française $\mathrm{n}^{\circ} 82$, Vers une didactique du français?, mai 1989, Paris, Larousse, pp. 45-52.

DABÈNE, Michel (1999) : Repères $\mathrm{n}^{\circ}$ 20, Recherches-actions et didactique du français. Hommage à Hélène Romian, Paris, INRP, 1999, pp. 37-41.

DAUNAY, Bertrand (2002) : «Le lecteur distant. Positions du scripteur dans l'écriture du commentaire ", Pratiques $\mathrm{n}^{\circ} 113-114$, Images du scripteur et rapport à l'écriture, Metz, CRESEF, 2002, pp. 135-153.

- (2007a) : «Comment la recherche didactique évalue les pratiques d'enseignement de la littérature », dans DUFAYS, J.-L. éd. (2007) Enseigner et apprendre la littérature aujourd'hui, pour quoi faire? Sens, utilité, évaluation, Louvain, Presses Universitaires de Louvain, pp. 35-43.

- (2007b) : Invention d'une écriture de recherche en didactique du français, Note de synthèse en vue de l'habilitation à diriger des recherches, Université Charles-de-Gaulle - Lille 3, mai.

DAUnAY, Bertrand, DUfAYS, Jean-Louis (2007) : « Méthodes de recherche en didactique de la littérature », Lettre de l'AIRDF n ${ }^{\circ} 40$, Namur, pp. 8-13.

DELCAMBRE, Isabelle, REUTER, Yves (2002) : « Images du scripteur et rapports à l'écriture », Pratiques ${ }^{\circ}$ 113-114, Images du scripteur et rapport à l'écriture, Metz, CRESEF, 2002, pp. 7-28.

DFLM (1998) : «Texte d'orientation de l'association », La lettre de la DFLM, $\mathrm{n}^{\circ} 23$, Lille, DFLM, pp. 28-31.

GAGné, Gilles, LAZURE, Roger, SPREnGER-ChAROLles, Liliane, Ropé, Françoise (1989) : Recherches en didactique et acquisition du français langue maternelle, Tome 1, Cadre conceptuel, thésaurus et lexique des mots-clés, Bruxelles, De Boeck-Wesmael, Paris, Editions Universitaire-INRP, Montréal, Université de Montréal-PPMF.

GolgOUX, Roland (2001) : «Recherche en didactique du français : contribution aux débats d'orientation », dans MARQUILló LARRUY, M. éd., Questions d'épistémologie en didactique du français (langue maternelle, langue seconde, langue étrangère), Université de Poitiers, pp. 125-132.

HALTÉ, Jean-François (1987) : «Écriture, littérature, formation », Les Cahiers du CRELEF $\mathrm{n}^{\circ} 25$, Questionnement pédagogique et texte littéraire, 1987--2, Besançon, CRELEF, pp. 81-103.

- (1992) : La didactique du français, Paris, PUF, collection «Que saisje ?»

- (2001) : « Des modèles de la didactique aux problèmes de la DFLM », dans MARQUILló LARRUY, M. éd., Questions d'épistémologie en didactique du français (langue maternelle, langue seconde, langue étrangère), Université de Poitiers, pp. 13-19.

— (2002) : «Didactique de l'écriture, didactique du français : vers la cohé- 
rence configurationnelle », dans Pratiques $n^{\circ} 115 / 116$, L'écriture et son apprentissage, Metz : CRESEF, pp. 15-28.

- (2005) : «Intégrer l'oral : pour une didactique de l'activité langagière », dans Halté, J.-F., Rispail, M.éd. (2005) L'oral dans la classe. Compétences, enseignement, activités, Paris, L'Harmattan, pp. 11-31.

Hameline, Daniel (2005) :, article «Pédagogie », dans CHAMPY, Philippe, ETÉVÉ, Christiane dir., Dictionnaire encyclopédique de l'éducation et de la formation, Paris, Retz.

Hofstetter, Rita, Schneuwly, Bernard (1998/2001) : « Sciences de l'éducation entre champs disciplinaires et champs professionnels », dans HOFSTETTER, R., SCHNEUWLY, B. éd., Le pari des sciences de l'éducation, Bruxelles, De Boeck (Raisons éducatives), pp. 7-25.

JAUBert, Martine, REBIÈre, Maryse, BERniÉ, Jean-Paul (2003) : « L’hypothèse "communauté discursive": d'où vient-elle ? où va-t-elle? », Les Cahiers Théodile $\mathrm{n}^{\circ} 4$, Villeneuve d'Ascq, Université Charles de Gaulle - Lille 3, pp. 51-80.

LAHANIER-REUTER, Dominique, Roditi, Éric dir. (2007) : Questions de temporalité. Les méthodes de recherche en didactiques (2), Villeneuve d'Ascq, Presses universitaires du Septentrion.

LAHIRE, Bernard (1993) : Culture écrite et inégalités scolaires. Sociologie de "l'échec scolaire » à l'école primaire, Lyon, Presses Uuniversitaires de Lyon.

LANGUE FRANÇAISE $\mathrm{n}^{\circ}$ 82, Vers une didactique du français ?, mai 1989, Paris, Larousse

MARquilló LARRUY, M. éd. (2001) : Questions d'épistémologie en didactique du français (langue maternelle, langue seconde, langue étrangère), Université de Poitiers.

Mercier, Alain, Schubauer-Leoni, Maria-Luisa, Sensevy, Gérard (2002) : "Vers une didactique comparée », Revue Française de Pédagogie n ${ }^{\circ}$ 141, Vers une didactique comparée, octobre-novembre-décembre 2002, Paris, INRP, pp. 5-16.

NONNON, Élisabeth (2000) : Les pratiques discursives dans la classe et les discours sur les pratiques : jugements de "qualitié », normes et polyphonie, Note de synthèse en vue de l'habilitation à diriger des recherches, Université Paris $\mathrm{V}$, décembre.

- (2006) : «Jean-François Halté au présent », Repères $\mathrm{n}^{\circ} 33$, La fiction et son écriture, Lyon, INRP, pp. 223-226.

PASSERON, Jean-Claude (1991/2006) : Le Raisonnement sociologique, Paris, Albin Michel.

PERrin-Glorian, Marie-Jeanne, Reuter, Yves dir. (2006) : Les Méthodes de recherche en didactiques, Villeneuve d'Ascq, Presses universitaires du Septentrion.

Petitjean, André (1998) : «La transposition didactique en français », Pratiques $\mathrm{n}^{\circ}$ 97-98, La transposition didactique en français, juin 1998, Metz, CRESEF, pp. 7-34.

Petitjean, André, Privat, Jean-Marie éd.(1999): Histoire de l'enseignement du 
français et textes officiels. Actes du colloque de Metz, Metz, Université de Metz, collection « Didactique des textes ».

REUTER, Yves (1995/2005) : «Synthèse. Didactique du français : éléments de réflexion et de proposition », dans CHISS Jean-Louis, DAVID, Jacques, REUTER, Yves dir., Didactique du français. Fondements d'une discipline, Bruxelles, De Boeck, pp. 211-234.

- (2006a): «Didactique et engagement(s)», Enjeux n ${ }^{\circ} 66$, Enseignement et engagement. Hommage à Jean-Maurice Rosier, Namur, CEDOCEF, pp. 715.

— (2006b) : «À propos des usages de Goody en didactique. Éléments d'analyse et de discussion ", Pratiques n 131-132, La littératie. Autour de Jack Goody, Metz, CRESEF, pp. 131-154.

- éd. (2007) : Dictionnaire des concepts fondamentaux des didactiques, Bruxelles, De Boeck.

Ropé, Françoise (1990) : Enseigner le français. Didactique de la langue maternelle, Paris, Éditions Universitaires.

ROSIER, Jean-Maurice (2002) : La didactique du français, Paris, PUF, collection «Que sais-je?».

Roulet, Eddy (1989) : " Des didactiques du français à la didactique des langues », Langue française ${ }^{\circ}{ }^{82}$, Vers une didactique du français ?, mai 1989, Paris, Larousse, pp. 3-7.

SCHNEUWLY, Bernard (1990) : «Didactique : quelques notes sur son histoire », La Lettre de la DFLM n ${ }^{\circ}$ 7, Paris, DFLM, pp. 22-24.

- (2007) : «Une histoire de ces 20 dernières années : Vygotski et les recherches en didactiques des disciplines ", colloque d'Albi, Vygotski et les recherches en éducation et en didactiques des disciplines.

SIMARD, Claude (2001) : «Bases épistémologiques de la didactique du français langue première ", dans MARQUILLÓ LARRUY M. éd., Questions d'épistémologie en didactique du français (langue maternelle, langue seconde, langue étrangère), Université de Poitiers, pp. 31-38.

VINCENT, Guy dir., (1994) : L'éducation prisonnière de la forme scolaire? Scolarisation et socialisation dans les sociétés industrielles, Lyon, Presses Universitaires de Lyon. 